\title{
Design and development of a thin film dielectric beam combiner
}

\author{
K V S R APPARAO and N K SAHOO* \\ Spectroscopy Division, MDRS*, Bhabha Atomic Research Centre, Trombay, Bombay \\ 400085 . India
}

\begin{abstract}
A multilayer thin film device called a beam combiner for a specific photodissociation experiment was designed and developed. The device when operated at $45^{\circ}$ angle of incidence transmits a UV laser beam and reflects a visible laser beam at the same time with minimum energy loss. The efficiency of the device was found to be better than $90 \%$.
\end{abstract}

Keywords. Thin films; beam combiner; edge filter; optics.

\section{Introduction}

Dielectric beam combiner is a multilayer thin film device which can be used to combine optical electromagnetic radiation of two different frequencies collinearly with minimum energy loss. The device has an application in a certain photodissociation process wherein the reaction target zone has to be irradiated collinearly with two laser beams within a few nanoseconds of delay. The wavelength of one laser is in the uv region and the other in the visible region. Since the efficiency of the dissociation process is proportional to the intensity of the laser radiation reaching the target, the device used should have minimum energy loss. Since no device of the required specifications was commercially available, a multilayer thin film device called a beam combiner was designed and developed for the specific application. Details involving the design and development of the beam combiner are briefly discussed in this paper.

\section{Design of the beam combiner}

The main requirements of the beam combiner were when operated at $45^{\circ}$ angle of incidence it should transmit $337 \mathrm{~nm} \mathrm{~N} \mathrm{~N}_{2}$ laser beam and at the same time reflect the tunable dye laser beam from $420 \mathrm{~nm}$ to $450 \mathrm{~nm}$ completely without any energy loss. These requirements can be achieved ideally with a $45^{\circ}$ angle of incidence low pass all dielectric edge filter having the edge anywhere between $350 \mathrm{~nm}$ and $380 \mathrm{~nm}$ and having about $150 \mathrm{~nm}$ to $200 \mathrm{~nm}$ wide high reflection zone.

A simple design of a conventional quarter wave stack with alternate low and high index films can be used as a beam combiner with low transmission efficiency. The reduction of the transmission in the uv region for such simple design is because of the presence of the characteristic ripple of the quarterwave stack in the pass region. The computed transmission (average of P-and S-polarized components) of such a simple design is shown in figure 1 (design $A$ ). The uv efficiency of the design can be increased by reducing the ripple in the pass band region from $300 \mathrm{~nm}$ to $400 \mathrm{~nm}$ by using matching 


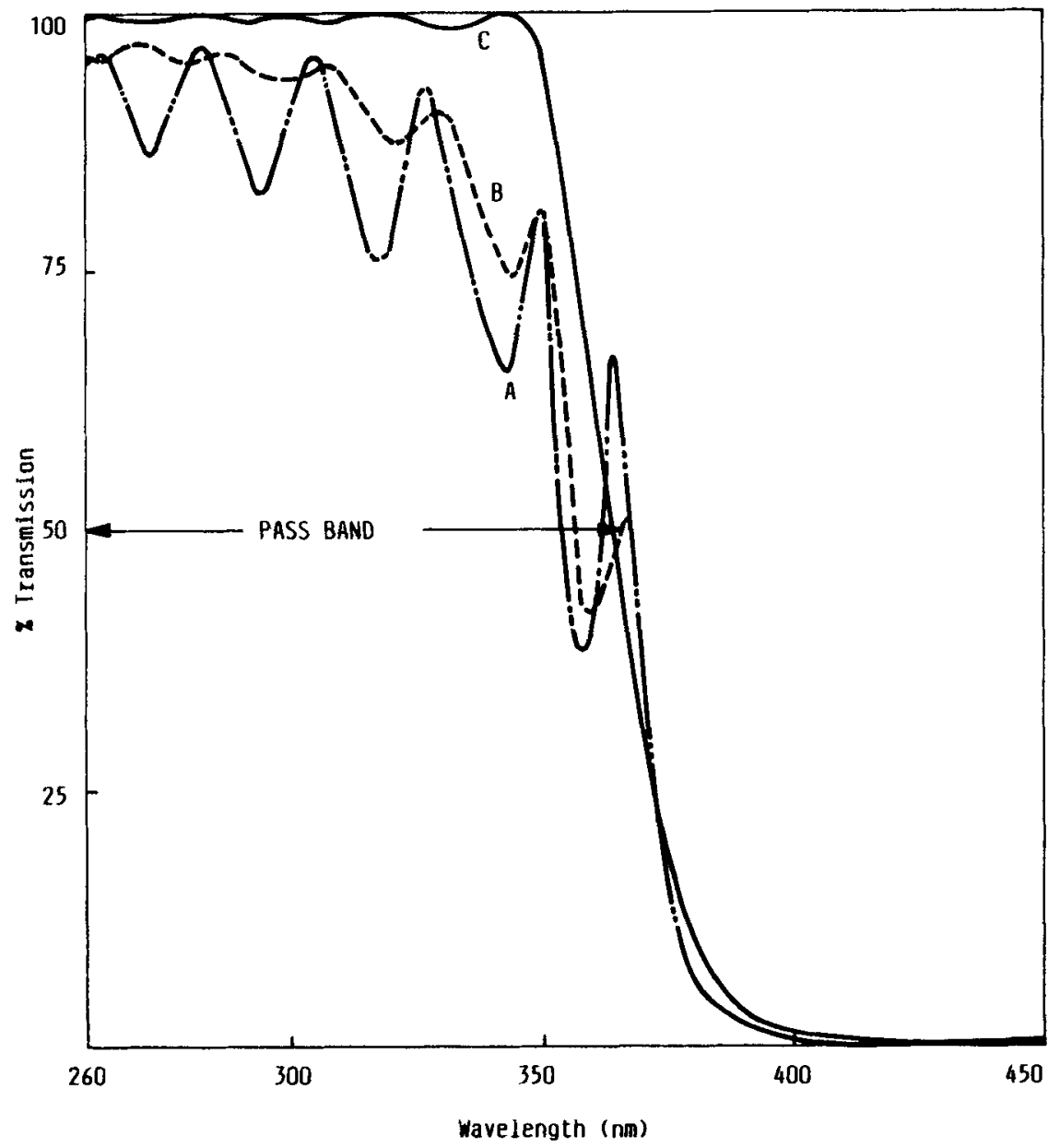

Figure 1. Transmission characteristic of beam combiner designs operating at $45^{\circ}$ angle of incidence. $\mathbf{A}, \mathbf{B}, \mathbf{C}$ refer to the respective designs.

techniques. The simplest approach for such design (Macleod 1969) is to add matching layers at either side of the quarterwave stack to match it to the substrate and to the medium. A quarterwave layer of admittance $\eta_{s}$ inserted between the stack and the substrate, another quarterwave layer of admittance $\eta_{1}$ between the stack and the medium according to the following relations becomes a good match

$$
\eta_{s}=\left(n_{s} \cdot E\right)^{1 / 2} \text { and } \eta_{1}=\left(n_{0} \cdot E\right)^{1 / 2}
$$

where $E$ is the equivalent optical admittance of the stack, $n_{s}$ the refractive index of the substrate and $n_{0}$ is that of the medium. These additional quarterwave layers at the required wavelength $(337 \mathrm{~nm})$ act as antireflection coatings in reducing the reflection ripple in the pass band. But in practice it is difficult to get suitable thin film materials having matching indices. The solution to the problem is then to have eighth wave thick low index layers on either sides of the stack which act as antireflection coatings to a first approximation. The transmission characteristic of such a 17-layer stack design is shown in figure 1 (design B). As seen in figure 1, design B has less ripple in the pass band 
compared to design A. To correct the ripple further in the pass band more advanced design methods have to be used. Using such an advanced method, a beam combiner was designed having less than $2 \%$ ripple in the pass band and its transmission characteristic is included in figure 1 (design $\mathrm{C}$ ). The design method used was based on a technique called "limited scan dampled least squares optimization", the details of which were described elsewhere (Apparao 1975 and 1977). The transmission characteristic of such an ideal beam combiner design is also shown in figure 1 (design $\mathrm{C}$ ). The design data for all the three designs are given in table 1 . Design $B$ which is relatively easy to develop and which serves the application is chosen for the present development.

\section{Development of the beam combiner}

The beam combiner according to design $B$ was developed and successfully fabricated by the method of vacuum evaporation using a conventional $300 \mathrm{~nm}$ vacuum coating plant. Optical grade $\mathrm{Sb}_{2} \mathrm{O}_{3}$ and $\mathrm{Na}_{3} \mathrm{AlF}_{6}$ were chosen as high and low index films respectively and were evaporated in boat sources. Fused silica substrate ( $50 \mathrm{~mm}$ dia) was used for the device. $\mathrm{Sb}_{2} \mathrm{O}_{3}$ films evaporated at a rate less than $5 \AA / \mathrm{sec}$ and $\mathrm{Na}_{3} \mathrm{AlF}_{6}$ films evaporated at about $20 \AA / \mathrm{sec}$ were found to give homogeneous stable films with minimum energy losses, particularly in the uv region. Optical and quartz crystal monitors were used during the evaporation time for monitoring optical thickness and rate of evaporation respectively. Computer-simulated data was used to monitor the film thicknesses. The performance of the 17-layer beam combiner thus fabricated was evaluated at the required wavelength points using lasers and power meter. The beam

Table 1. Optical description of 17 laser beam combiner designs

\begin{tabular}{|c|c|c|c|c|}
\hline \multirow{2}{*}{$\begin{array}{l}\text { No. of } \\
\text { layers }\end{array}$} & \multirow{2}{*}{$\begin{array}{c}\text { Ref. } \\
\text { index }\end{array}$} & \multicolumn{3}{|c|}{ Optical thickness of the film in $\mathrm{nm}$} \\
\hline & & Design A & Design B & Design $C$ \\
\hline & 1.0 & Massive & Massive & Massive \\
\hline 1 & 1.35 & $120 \cdot 0$ & $60 \cdot 0$ & $65 \cdot 13$ \\
\hline 2 & $2 \cdot 30$ & $120 \cdot 0$ & $120 \cdot 0$ & $126 \cdot 32$ \\
\hline 3 & 1.35 & $120 \cdot 0$ & $120-0$ & $124 \cdot 03$ \\
\hline 4 & $2 \cdot 30$ & $120 \cdot 0$ & $120 \cdot 0$ & 122.45 \\
\hline 5 & $1 \cdot 35$ & $120 \cdot 0$ & $120 \cdot 0$ & $122 \cdot 45$ \\
\hline 6 & $2 \cdot 30$ & $120 \cdot 0$ & $120 \cdot 0$ & $119 \cdot 70$ \\
\hline 7 & $1 \cdot 35$ & $120 \cdot 0$ & $120 \cdot 0$ & $118 \cdot 23$ \\
\hline 8 & $2 \cdot 30$ & $120 \cdot 0$ & $120 \cdot 0$ & $118 \cdot 52$ \\
\hline 9 & $1 \cdot 35$ & $120 \cdot 0$ & $120 \cdot 0$ & $119 \cdot 11$ \\
\hline 10 & $2 \cdot 30$ & $120 \cdot 0$ & $120 \cdot 0$ & $119 \cdot 11$ \\
\hline 11 & 1.35 & $120 \cdot 0$ & 1200 & 118.81 \\
\hline 12 & $2 \cdot 30$ & $120 \cdot 0$ & 1200 & $119 \cdot 40$ \\
\hline 13 & 1.35 & $120 \cdot 0$ & $120 \cdot 0$ & 120.91 \\
\hline 14 & $2 \cdot 30$ & $120 \cdot 0$ & $120 \cdot 0$ & 123.08 \\
\hline 15 & 1.35 & $120 \cdot 0$ & $120 \cdot 0$ & 125.98 \\
\hline 16 & $2 \cdot 30$ & $120 \cdot 0$ & $120 \cdot 0$ & 134.08 \\
\hline \multirow[t]{2}{*}{17} & 1.35 & $120 \cdot 0$ & $60 \cdot 0$ & 156.35 \\
\hline & 1.48 & Massive & Massive & Massive \\
\hline
\end{tabular}


combiner when operated at $45^{\circ}$ angle of incidence showed more than $90 \%$ transmission at $337 \mathrm{~nm}\left(\mathrm{~N}_{2}\right.$ laser) and more than $99 \%$ reflection in the region from $420 \mathrm{~nm}$ to $450 \mathrm{~nm}$ (tunable dye laser). The device is being used successfully in the photodissociation experiments in our laboratories.

\section{References}

Apparao K V S R 1975 Indian J. Pure Appl. Phys. 13183

Apparao K V S R 1977 Indian J. Pure Appl. Phys. 15270

Macleod H A 1969 in Thin film optical filters (London: Adam Hilgers) p. 128 